\title{
Alzheimer's disease: diagnostics, prognostics and the road to prevention
}

\author{
Iris Grossman • Michael W. Lutz • \\ Donna G. Crenshaw • Ann M. Saunders • \\ Daniel K. Burns • Allen D. Roses
}

Received: 4 March 2010 /Accepted: 19 May 2010 /Published online: 29 June 2010

(C) The Author(s) 2010. This article is published with open access at Springerlink.com

\begin{abstract}
Alzheimer's disease (AD) presents one of the leading healthcare challenges of the 21st century, with a projected worldwide prevalence of $>107$ million cases by 2025. While biomarkers have been identified, which may correlate with disease progression or subtype for the purpose of disease monitoring or differential diagnosis, a biomarker for reliable prediction of late onset disease risk has not been available until now. This deficiency in reliable predictive biomarkers, coupled with the devastating nature of the disease, places $\mathrm{AD}$ at a high priority for focus by predictive, preventive and personalized medicine. Recent data, discovered using phylogenetic analysis, suggest that a variable length poly-T sequence polymorphism in the TOMM40 gene, adjacent to the $A P O E$ gene, is predictive of risk of $\mathrm{AD}$ age-of-onset when coupled with a subject's current age. This finding offers hope for reliable assignment of disease risk within a 5-7 year window, and is expected to guide enrichment of clinical trials in order to speed development of preventative medicines.
\end{abstract}

Keywords Alzheimer's disease - Personalized medicine . Phylogenetic analysis - Translocase of outer mitochondrial membrane (TOMM40) - Apolipoprotein E · Poly-T variant

\author{
I. Grossman · D. K. Burns · A. D. Roses \\ Cabernet Pharmaceuticals, \\ Durham, NC, USA \\ M. W. Lutz • D. G. Crenshaw • A. M. Saunders • D. K. Burns • \\ A. D. Roses $(\bowtie)$ \\ Duke University, \\ Box 90344, Durham, NC 27708-0120, USA \\ e-mail: allen.roses@duke.edu \\ M. W. Lutz • D. G. Crenshaw • A. M. Saunders · D. K. Burns \\ A. D. Roses \\ Deane Drug Discovery Institute, \\ Durham, NC, USA
}

\section{"An ounce of prevention is worth a pound of cure"}

The ultimate goal of modern healthcare is to reduce the burden and costs associated with disease risk, monitoring and treatment by utilizing prevention programs in a costeffective manner. While it is considered common sense that "an ounce of prevention is worth a pound of cure", there are few examples where active intervention strategies have been successfully and systematically developed, with the obvious exception of vaccines. The paucity of prevention therapies is not surprising, given the high benefit-risk ratio required when administering active drugs to (currently) healthy individuals. It is therefore critical to develop diagnostics that pinpoint the indicated subpopulation for which the benefit-risk profile is maximized and the intervention is clearly justified. Such diagnostics need to demonstrate clinical utility, as well as rigorous technical performance characteristics. The decisive test of such a diagnostic hinges on its proven predictive value characteristics, which would then be adopted into routine use by practicing physicians and healthcare insurers. Therefore, this review first evaluates the characteristics of clinically useful diagnostics in general, and then focuses on genetic markers and diagnostics for Alzheimer's disease (AD). Finally, we describe a novel genetic marker for AD and discuss its application in the design of a prevention/delay of disease onset clinical trial that may directly impact medical practice.

\section{Properties of clinically useful biomarkers}

What transforms a biomarker into a clinically useful diagnostic? Evidence-based medicine guidelines outline a sequence of steps that establish the Analytical validity, Clinical validity, 
Clinical utility and Ethical, legal and social aspects (ACCE) of diagnostic and prognostic tests of a biological or genomic nature $[1,2]$. The evidence gathered throughout the ACCE process should be conducted according to appropriately documented good operational practices with respect to sample collection, storage and handling. The initial and most standardized step requires demonstration of high sensitivity, specificity and reproducibility of the test's results, in order to ensure its technical accuracy in capturing the targeted genetic polymorphisms. Once the analytical performance of the test is validated in vitro, the biomarker is tested for clinical validity versus endo-phenotypes or quantitative clinical endpoints. At this stage, the performance of the diagnostic is tested for sensitivity, specificity and reproducibility in accurately measuring the clinically relevant end-points. Clinical validity can be studied retrospectively, using previously collected samples, as long as the appropriate samples were collected from the vast majority of cohort participants with the appropriate informed consent.

The third component of the guidelines focuses on clinical utility and is the ultimate test of impact of the diagnostic on health outcomes. While during the course of clinical validity testing the value of the diagnostic is projected based on technical performance and market estimates, the "clinical utility" step is designed to test these hypotheses in the context of healthcare management. This has become a pivotal component of clinical decision making, whether as part of a drug development pipeline or in the context of medical practice. Clinical utility studies are designed to optimize testing conditions and quantify the predictive value of the novel diagnostic in the context of controlled clinical settings. Once validated, further studies may be conducted to assess cost-effectiveness and adoption characteristics in real-world clinical settings and may employ comparative effectiveness models in order to weigh the novel, biomarker-integrated treatment algorithms against standard-of-care [3]. Other aspects that need to be considered in addition to demonstrating validity and utility of a biomarker include Ethical, Legal and Social Issues (ELSI) that arise when introducing the diagnostic to the market. Eventually, the success of a diagnostic relies on the successful implementation of each of the above ACCE model components [2], as reflected via endorsement by regulators, incorporation into practice guidelines, influence on physician behaviors and adoption into reimbursement policies [4].

A direct method for demonstrating the most significant component of the ACCE framework - the clinical utility of a biomarker - utilizes a prospective study design in parallel to a drug development trial. To this end, the FDA issued a concept paper that proposes a framework for the codevelopment of drugs and diagnostics via an integrated combination regulatory path [5]. A pioneering example for drug/diagnostic $(\mathrm{Rx} / \mathrm{Dx})$ co-development in the $\mathrm{AD}$ therapeutic area is outlined in the following sections.

\section{The challenge of Alzheimer's disease management}

$\mathrm{AD}$ has become an area of considerable unmet medical need in recent decades. Currently, over 5 million Americans, or more than $10 \%$ of citizens 71 years of age or older, suffer from $\mathrm{AD}$ and estimates are that 1 new case of $\mathrm{AD}$ develops every 7 seconds, with a higher rate of increase in developing countries $[6,7]$. Unless effective therapies for preventing or delaying $\mathrm{AD}$ onset are developed, it is projected that $>16$ million Americans and $>107$ million people worldwide will suffer from AD by 2050. Delaying AD onset by as few as one or two years is speculated to decrease the worldwide disease burden in 2050 by 12 million or 23 million cases, respectively [8]. In addition to the devastating losses of quality of life and productivity experienced by AD patients, their care-givers spend greater than $\$ 94$ billion annually (last updated in 2004) in the US on AD-related healthcare costs [9]. These numbers are expected to increase substantially with the anticipated growth in both population and life expectancy, particularly as the "baby-boom" generation ages. The increased burden, however, cannot be offset by effective medical management using currently available AD healthcare strategies. There are currently no longterm, effective treatments for $\mathrm{AD}$, and no life-style changes have been proven to substantially delay or prevent its onset [10].

Why does $A D$ present such a formidable challenge to healthcare management and novel drug discovery? $\mathrm{AD}$ is a neurological disorder that presents as decline in domains of cognition, memory and activities of daily living. The typical pattern of memory loss involves impaired recall of learned or previously known information, reflecting medial temporal lobe (and possibly basal forebrain) dysfunction. While overlapping, this pattern is distinct from normal aging, where learning efficiency, working memory and psychomotor speed (activities related to frontal lobe function) are reduced [11]. The two histopathological hallmarks of AD brains are: (1) intracellular neurofibrillary tangles (NFT) resultant from the aggregation of hyperphosphorylated tau proteins; and (2) extracellular senile plaques, composed primarily of amyloid beta $(A \beta)$ protein. Unfortunately, clinical characterization of the disease, through impaired behavioral autonomy or learning and cognition deficits, is complex, subjective and includes a range of possible differential diagnoses, deeming definitive $\mathrm{AD}$ diagnosis contingent on autopsy. The gradual progression of the disease has allowed researchers to identify an intermediate phenotype entitled mild cognitive impairment (MCI) that may allow for early diagnosis and development of predictive and preventive models targeted at arresting or delaying the progression to $\mathrm{AD}$ stages. By focusing research efforts on the phenotypic definition of MCI, researchers may eventually be able to prevent or delay conversion into $\mathrm{AD}$ in this at-risk population. However, it is important to note that 
the annual rate of $\mathrm{AD}$ diagnosis among MCI subjects is only $10-15 \%$. Moreover, individuals with MCI tend to develop $\mathrm{AD}$, but they may also develop other forms of non-AD types of dementia, such as frontotemporal dementia (FTD). MCI patients can also maintain a steady level of impairment without any progression in severity [12]. Overall, the late onset of $\mathrm{AD}$ and its intermediate phenotype, the heterogeneous presentation patterns and the sporadic manifestation make research, diagnosis and prognosis challenging.

\section{Genetic markers of Alzheimer Disease}

Genetic diagnostics measure DNA polymorphisms that are associated with biological or clinical traits. Genetic markers originating from germ line polymorphisms (as opposed to somatic mutations) can be detected in samples of blood, saliva or differentiated non-cancerous tissues at any timepoint because they are static in nature. Such measurements can serve as predictive diagnostics early on in life, and when combined with other dynamic markers (such as serum protein levels or even age) may provide reliable and accurate composite scores for probability of disease risk, progression or treatment response within a short window of time. Such composite predictors are expected to contribute substantially to the development of medical management guidelines [13].

$\mathrm{AD}$ has a strong genetic component, with up to $80 \%$ heritability as estimated from twin-concordance studies [14]. The disease is roughly categorized into two main subtypes: 1) autosomal dominant, early onset AD, defined by disease presentation before the age of 61 , is accounted for by familial AD (FAD) and 2) "sporadic" (non-familial) $\mathrm{AD}$, most commonly termed late onset AD (LOAD), where symptomatic presentation generally starts after the age of 55 . For research purposes LOAD is usually defined as $>60$ years of age to avoid overlap with the familial early-onset forms. Most of the successes registered for AD genetic research have been reported for the Mendelian forms of the disease, the autosomal dominant FAD. Rare mutations have been identified in three different (yet biologically associated) genes: amyloid precursor protein $(A P P)$ [15], presenilin-1 (PSEN1) [16], and presenilin-2 (PSEN2) [17, 18]. However, FAD is rare and accounts for approximately $10 \%$ of earlyonset $\mathrm{AD}$ and less than $1 \%$ of all AD cases [19]. The most common form of $\mathrm{AD}$ is LOAD [20], and the only firmly established genetic risk factor for LOAD is the $E 4$ allele of the $A P O E$ gene [21], as detailed below. Collectively, genetic variations in $A P P, P S E N 1, P S E N 2$ and $A P O E$ are estimated to account for only $25-50 \%$ of the overall genetic risk for $A D$ $[22,23]$. It is therefore clear that other susceptibility genes remain undiscovered, including the possibility of finding a single genetic variation with a higher positive predictive value.

\section{$A P O E$ and Alzheimer's disease}

The apolipoprotein E (APOE) gene encodes a major apolipoprotein that is synthesized chiefly in the liver, but also in a range of other organs, including the brain [24]. The protein product, apoE, acts primarily as a transporter of cholesterol and lipids among various cells of the body, but it has also been proposed to serve other important functions in both physiology and pathology [24]. The discovery of $A P O E$ 's involvement in LOAD and the identification of the $E 4$ at-risk allele were first reported in 1993 [21, 25]. It is important to note, that the discovery of the association between sequence variations in the $A P O E$ gene and clinically meaningful variability in $\mathrm{AD}$ risk pinpointed the pivotal role that apoE plays in the pathogenesis of $A D$, which had not been fully explored beforehand. In fact, apart from age, APOE4 status is the most validated AD risk factor. Conversely, the rare $E 2$ allele has been associated with protection against LOAD compared with both the $E 4$ allele and the more common E3 allele [21]. Overall, it has been reported that heterozygotes for the $E 4$ allele are three to four times as likely to develop AD than non-carriers [26], and the number of copies (one or two alleles) of $E 4$ carried is proportional to level of risk [27, 28]. As seen in the initial reported finding depicted in Fig. 1 [28], the Kaplan-Meier survival graph shows a characteristic curve for each one of the genotype carrier groups: the number of $E 4$ copies carried is significantly associated with disease manifestation age, with $E 4 / E 4$ carriers showing the earliest disease onset. This association of $A P O E 4$ with the lower age of disease onset, in addition to its association with overall disease risk, has also been repeatedly confirmed by independent groups [21, 29-35].

Functional research heralded by the 1993 discovery has identified multiple pathways in which apoE is involved in LOAD pathoetiology [36]. Indeed, abnormality in these processes has been shown to associate with the sequence, structure and biochemical distinctions between E4 and E3 or E2 alleles. In fact, apoE4 is the only molecule that has to-date been associated with the entire spectrum of biochemical disturbances characteristic of $\mathrm{AD}$ : $\mathrm{A} \beta$ deposition, tangle formation, oxidative stress, lipid homeostasis deregulation, synaptic plasticity loss and cholinergic dysfunction [36, 37]. Given the fact that the APOE4 allele frequency is quite high ( 0.15 in Caucasians [38]), asymptomatic carriers have long been studied as a convenient and informative AD research model. In this way, AD-like structural and physiological changes have been discovered and shown to exist years before the symptomatic onset of memory loss and dementia [39]. This has been a key component in the discovery and development of disease biomarkers, including neuroimaging. However, the usefulness of these biomarkers and $A P O E$ testing in clinical practice does not meet clinical utility criteria due to insufficient sensitivity, specificity and cost-effectiveness [40]. 


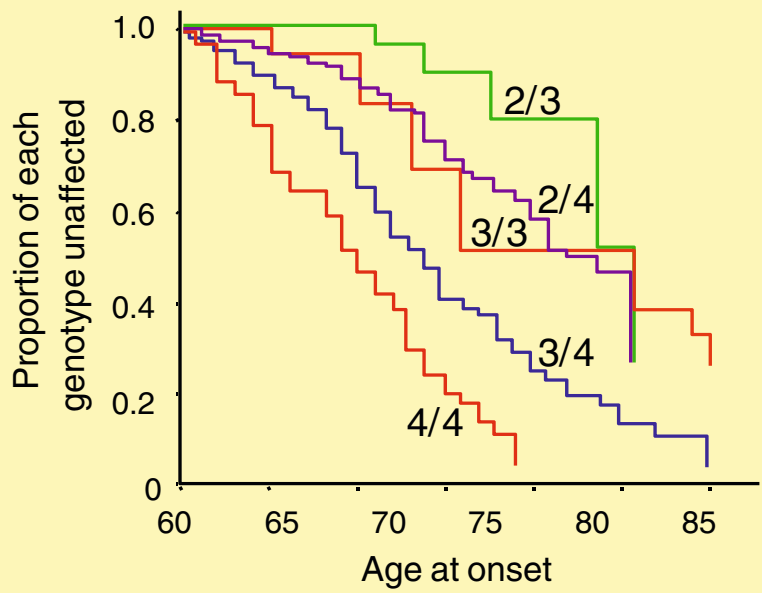

Fig. 1 Alzheimer's disease age of onset curves by $A P O E$ genotype, based on the information available in 1994 (adopted from [28]). Age of onset for LOAD, sporadic AD and control subjects as published in 1994 [28]. The age-of-onset is scored as a function of the individual's $A P O E$ genotype. Onset curves were estimated by Kaplan-Meier product limit distributions

Due to this fact and to the lack of preventative or curative measures many believe that prediction in healthy individuals is unethical and the consensus recommendation discourages genetic testing outside research settings.

\section{Genome-wide association studies of Alzheimer's disease}

Genome-wide association studies (GWAS) use screening technology to test a pre-defined set of common single nucleotide polymorphisms (SNPs) and large copy number variations (CNVs) across the entire genome against various phenotypic traits in a statistical association model. Overall, GWAS conducted in large cohorts have proven successful in suggesting a number of genetic variants of moderate and small effect sizes for complex diseases, including neurological disorders such as schizophrenia, autism spectrum disorders and $\mathrm{AD}[41,42]$. These reports have been most useful in confirming discoveries made in the last few decades based on linkage and candidate gene studies and have suggested novel regions of interest for further in-depth research, as has been successfully demonstrated in the field of diabetes susceptibility research, particularly with respect to TCF7L2 $[43,44]$. However, several limitations hinder the translation of GWAS findings from bench to bedside: (1) these technologies screen binary SNPs that serve also as proxies for some large CNVs, but are less suitable for the study of other smaller structural variation (e.g. polymononucleotide tracts, trinucleotide repeats, small insertion/deletions, etc); (2) markers selected for inclusion in these off-the-shelf high- throughput chips are based on co-occurrence probability (i.e. Linkage Disequilibrium, LD) and common allele frequency (5\% or higher for the most part). As a result, polymorphisms with low frequency and high LD to a common SNP are excluded. However, it is becoming evident that often these less common polymorphisms are the major, if not sole, source of association signal [45] (see further discussion below); (3) coverage of regions is generally even across the genome, but specific candidate genes of interest are often insufficiently represented; (4) rare, yet functionally validated markers in candidate genes of interest are often not included; (5) statistical approaches focus mainly on single markers, yielding results that are less robust due to multiple testing. Moreover, the effect sizes generally reported for common disease GWAS are marginal and their projected clinical utility has been particularly disappointing [46]. In summary, GWAS serve to highlight blocks of genetic regions of interest for association research. However, the pinpointing of causal variants, gene-gene and gene-environment interaction effects warrants increased resolution of the genetic variation within a candidate gene region, as well as more powerful and biologically plausible approaches to their analysis [47].

Since the association of $A P O E$ with $\mathrm{AD}$ was discovered, nearly 1,000 papers have been published reporting and refuting associations of $\mathrm{AD}$ with hundreds of genetic variants in different genes [42]. Recently, a meta-analysis study suggested that no more than a dozen reproducible associations between genomic regions and $\mathrm{AD}$ risk have actually been found [42]. Furthermore, about a dozen GWAS of sporadic LOAD have been published to date. The papers confirm that markers in the vicinity of $A P O E$ represent the major susceptibility genomic region for the disease. As illustrated in Table 1, no other AD association in the human genome remotely approaches the same level of statistical significance or effect size as the genomic region containing $A P O E[29,30,33,48-56]$. It should be emphasized that the two SNP determinants for $A P O E$ genotype are not included on the commercial platforms commonly used for GWAS, and the major SNPs that have extraordinarily statistical significance in the AD GWAS publications are located in the TOMM40 gene region and not in $A P O E$. In other words, although findings are usually referred to in the literature as associated with the $A O P E \mathrm{LD}$ region, the measurements commonly defining this region are rather TOMM40 polymorphisms.

\section{TOMM40 variable length polymorphism: increasing diagnostic prediction power and enabling a disease prevention trial}

The hypothesis that abnormal energy metabolism is a fundamental feature of $\mathrm{AD}$ pathology was proposed in the 90's [28, 
57-59]. Indeed, cerebral metabolic rate abnormalities precede any evidence for functional impairment by neuropsychological testing or for brain atrophy as seen in neuroimaging by several decades [60]. Evidence is amassing to support the hypothesis that defective mitochondrial metabolism and function is the instigating event of pathological changes that result in $\mathrm{AD}$ [61]. For instance, mitochondrial dysfunction in $\mathrm{AD}$ brains correlates with accumulation of A $\beta$ [62-64] and APP [65], as well as presence of apoE4 fragments, in mitochondria, causing loss of dynamic functions resulting in decreased synaptodendritic regeneration and subsequent neuronal cell death $[65,66]$. Deposition of these proteins is also associated with decreased glucose and oxygen utilization [67, 68]. Furthermore, it has been shown that in the mitochondrion APP forms stable complexes with the translocase of the outer mitochondrial membrane 40 (TOM40) import channel [69], and that the transport of $A \beta$ into the mitochondrion is mediated by the same TOM40 pore, further promoting neurotoxicity.

The significant genetic finding discussed in detail by Roses et al. [70] and outlined below is that a variable-length, deoxythymidine homopolymer (poly-T) in the gene encoding TOM40 (TOMM40) is associated with age-of-onset of $\mathrm{AD}$, the major endo-phenotype of the disease. It is critical to note that the TOMM40 gene is adjacent to, and in high LD with, $A P O E$, and the evolutionary relationship between the two genes inherited together on the same strand dictates that some specific TOMM40 variants are uniquely associated with each of the $A P O E$ alleles. $A P O E 4$ alleles are linked to long poly-T variants of the rs 10524523 locus approximately $98 \%$ of the time, while phylogenetic analyses clearly demonstrate a separate genetic origin of APOE3 strands, linked to either very long or short poly-T variants. Thus, a poly-T variant in the TOMM40 gene sub-divides $A P O E 3$ variants into high risk (very long) alleles and low risk (short) alleles, challenging the commonly accepted hypothesis that $A P O E 3$ is $\mathrm{AD}$ risk-neutral. This finding explains the current heterogeneity in $\mathrm{AD}$ age-of-onset registered within $A P O E 3$ carriers (Fig. 1), and offers the potential to predict the 5-7 year risk of disease onset in individuals carrying the $A P O E 3 / 3$, $A P O E 3 / 4$ and $A P O E 4 / 4$ genotypes, making up $~ 85 \%$ of all
AD cases. Additionally, the functional relevance of genetic polymorphisms in the TOMM40 gene region has been discussed recently: First, a combined set of three SNPs within the TOMM40 gene, one $A P O E$ promoter SNP and two SNPs within distal $A P O E$ enhancer elements was recently reported to predict CSF $A P O E$ levels [71]. Second, overlapping or linked SNPs spanning the entire $A P O E$ and TOMM40 region were reported to be associated with sporadic AD case-control analyses in all published GWAS with extraordinarily significant $p$-values, as clearly demonstrated in Table $1[29,49-54]$. The three SNPs with the highest significance were all from TOMM40.

The TOMM40 finding was enabled by a research approach that combined both intensive high resolution Sanger sequencing and the employment of phylogenetic statistical methodology. This combined strategy is likely to be successful in the study of other traits for which heritability has been shown to be high but the complex genetic architecture leading to, or modifying, the disease phenotypes hinders the effectiveness of conventional statistical and technological approaches. Similar approaches to the one employed herein are routinely used to study the evolution of simpler genomes, such as those of viruses and bacteria, and are particularly useful in annual vaccine production against flu viruses. However, it had not been commonly used as a method for complex disease susceptibility investigations, due to the size and complexity of the human genome. Currently the method is only practical if there are other indications that a particular genomic locus or region is involved in the phenotype of interest. In order to maximize the power of the method and our ability to discover the "missing heritability" residual to the $A P O E 4$ carriage effect, the entire region spanning the $A P O E$ TOMM40 genes was interrogated by deep Sanger sequencing. In other words, sequencing was performed in long DNA stretches that permitted incontrovertible assembly of co-occurrence combinations (also termed haplotypes) with specific $A P O E$ alleles. Deep sequencing also reliably detected small repeat elements such as poly-T variants. The resultant polymorphic map of common and rare, binary and multivariate polymorphisms was then analyzed using phylogenetic methods.

About a dozen GWAS of sporadic LOAD have been published to date. The papers
confirm that markers in the vicinity of $A P O E$ represent the major susceptibility genomic
region for the disease. As illustrated in Table 1 , no other AD association in the human
genome remotely approaches the same level of statistical significance or effect size as the
genomic region containing APOE. It should be emphasized that the two SNP determinants
for $A P O E$ genotype are not included on the commercial platforms commonly used for
GWAS, and the major SNPs that have extraordinarily statistical significance are located
in the TOMM 40 gene region and not in $A P O E$. In other words, although findings are
usually referred to in the literature as associated with the $A O P E$ LD region, the
measurements commonly defining this region are rather TOMM4O polymorphisms. 


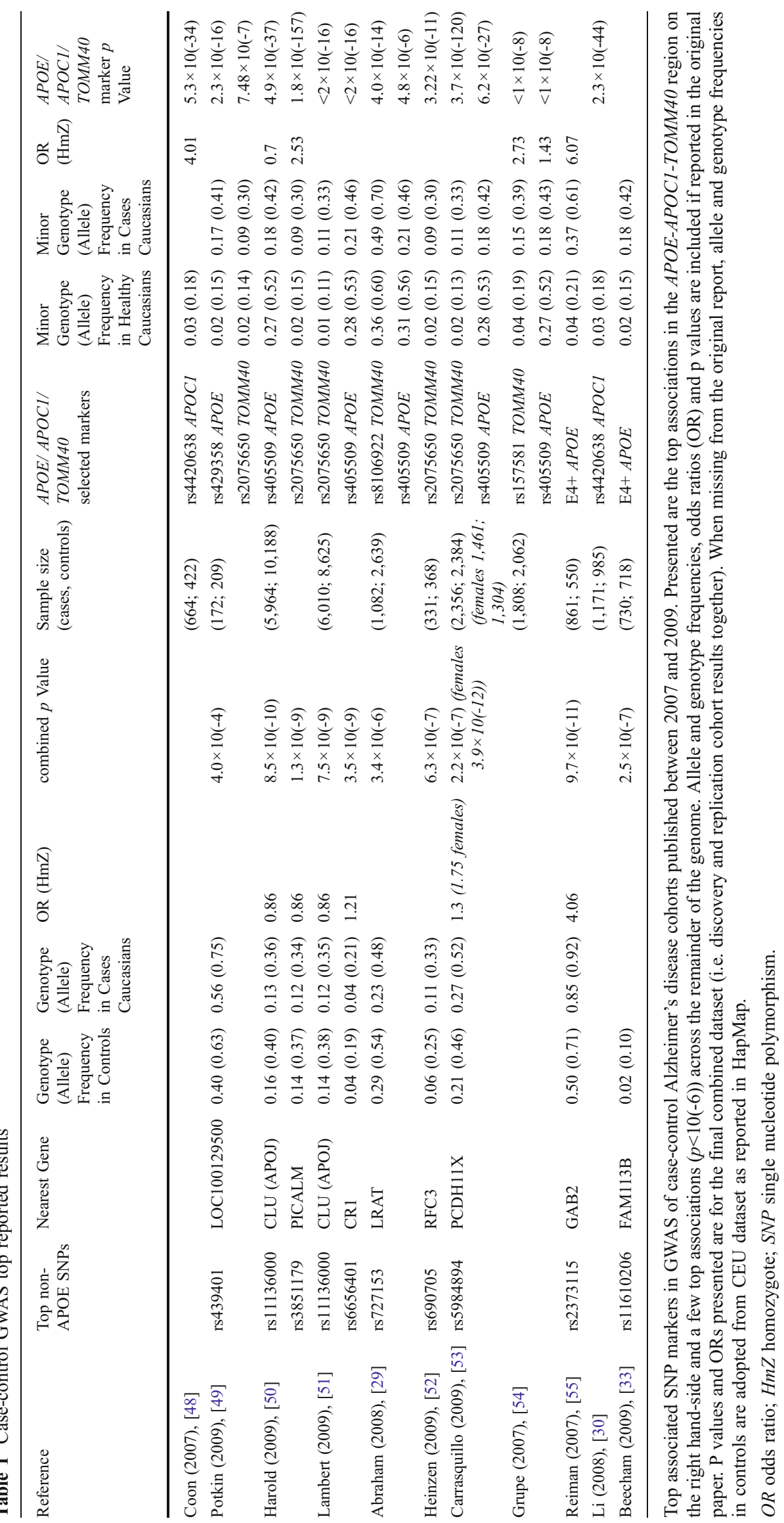


Phylogenetic (or genealogical) methods define the rare and common genetic variants on each strand of DNA in a region of interest, often selected based on its LD properties. This strategy significantly decreases the number of tests for an association study by clustering haplotypes according to sequence similarity. This similarity is assigned based on inference of the ancestral relationships between the various variants. Once variants are assigned into initial groups (referred to as clades), an iterative procedure of inferring subgroupings is employed until all variants are interconnected into a tree-like spatial structure. This overall process of tracking back the sequence of events, from present genetic make-up to ancestral configuration, and the phasing of polymorphisms into a branching tree structure is termed "coalescence". The assumption is that, due to patterns of inheritance, haplotypes that contain 'causal' variants will be more related to each other than to haplotypes that do not contain the causal variant(s) and will therefore cluster into a distinct clade(s). These risk haplotypes will therefore be embedded into the tree resultant from the analysis [72]. In this fashion, it is possible to shrink the overall set of polymorphisms present in a genomic region (often amounting to dozens or hundreds) into those that distinguish between "disease associated" clades and "control associated" clades. Thus, it is easily possible to reduce the complexity, multiplicity and computational burdens typical of conventional association methodology.

The discovery of the LOAD poly-T variant (rs10524523) was based on a series of experiments conducted sequentially in Caucasian cohorts, so as to discover genetic variants of interest in one cohort and confirm the results in independent datasets. Full details of the discovery and its confirmation, and the study designs that led to the identification of the specific genetic variants, were published elsewhere [70]. Overall, these data suggested that a significant genetic component of $\mathrm{AD}$ risk was due to the effect of a codominant variable repeat variant in TOMM40, acting cis to $A P O E 3$ and modifying the function of TOMM40 and/or $A P O E$ genes or gene products in some way that impacts onset of AD. Cis-regulatory elements and structural variants have previously been implicated in human evolution, variation, disease susceptibility and complex traits, so this outcome is not without precedence [73, 74]. In fact, it has been shown that even few mononucleotide repeat differences can alter gene expression in yeast [75] and humans [76], as well as modify RNA splicing in mice and humans [77]. Perhaps the most famous example of a poly$\mathrm{T}$ tract and its involvement in human disease has been reported for Cystic Fibrosis. The number of $\mathrm{T}$ residues present in the intron8-exon9 junction in the cystic fibrosis transmembrane conductance regulator (CFTR) gene affects splicing efficiency, resulting in reduced amounts of functional transcripts, which in turn lead to variable Cystic Fibrosis symptoms [78].

The discovery of the genetic variants responsible for the majority of variability in age-of-onset of LOAD in itself does not constitute any benefit to the clinical management of the disease. In order to prove the clinical validity and demonstrate clinical utility tailor-designed prospective clinical trials need to be conducted. To this end, a clinical trial is being designed, aiming to test the clinical diagnostic validity of rs10524523 (with or without APOE subtyping) as a predictor of $\mathrm{AD}$ risk and onset in a prospective fashion. The underlying premise of the study is that the TOMM40 rs10524523 marker, when modeled together with $A P O E$ subtype and age will translate into a more precise prediction of risk at a particular age than predictions based on $A P O E$ subtype alone. This should be reflected in improved resolution of the various age-of-onset Kaplan-Meier curves seen in Fig. 1. In particular, overlapping of the non-E4/E4 carrier curves in Fig. 1, may be due to low sample sizes of the less common genotype-carrier groups. Alternatively, the overlap may suggest the existence of additional important factors, such as other genetic variants or environmental factors that are involved in the development of the disease but are unevenly distributed across non- $E 4 / E 4$ carriers. It is hypothesized, and remains to be proven in the planned prospective validation study, that the separation between Kaplan-Meier curves will improve based on the composite TOMM40-APOE diagnostic, mainly due to redefinition of the APOE3-carrier curves according to their haplotypic relationship to rs10524523 (Fig. 2). The study design testing this hypothesis presents a pioneering $\mathrm{Dx} / \mathrm{Rx}$ codevelopment approach: the predictive diagnostic validity study is conducted simultaneously with a prevention (delay of onset) clinical trial employing a therapeutic agent in unaffected individuals (http://www.opalstudy.org). Further details on the possible design of this combination trial as discussed at the FDA's Voluntary Exploratory Data Submission discussions in October 2009 are detailed elsewhere [79].

\section{Diagnostic utility: genetic and other biomarkers}

Plasma, CSF and neuroimaging biomarkers of $\mathrm{AD}$ have been developed to serve as important surrogates of dynamic changes characteristic of $\mathrm{AD}$ progression [59, 80, 81]. They have been useful in research settings as means to measure protein-aggregation in plaques, to indicate abnormalities in metabolism of APP or to pinpoint neuronal degeneration. Much progress in understanding pathological processes at early and presymptomatic stages can be gained by recording changes in hippocampal volume and glucose metabolism. However, these dynamic biomarkers have 


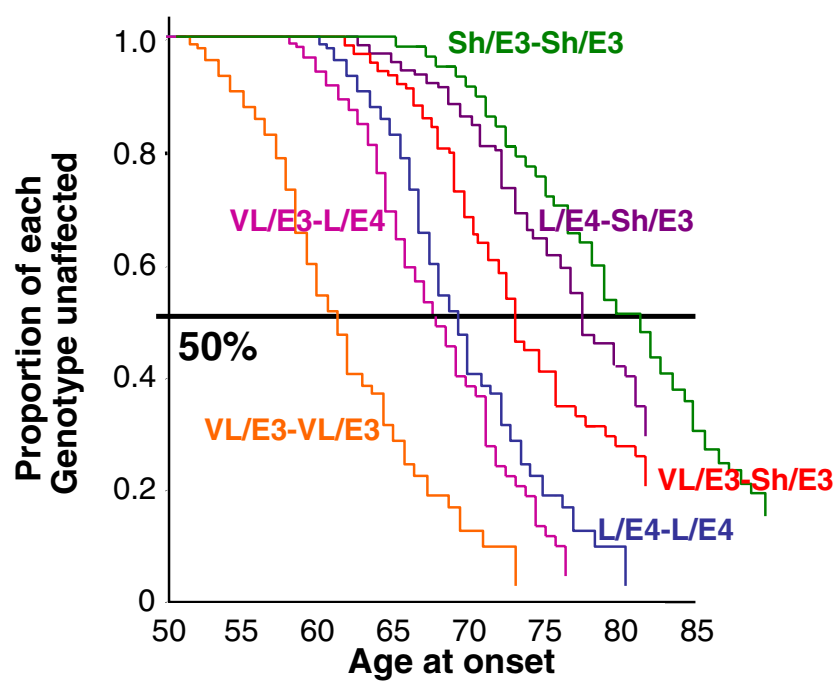

Fig. 2 Hypothetical Alzheimer's disease age of onset curves by TOMM40-APOE haplotype, based on the information available today (February 2010). We propose that each of the original AD age of onset curves is in fact a composite of sub-curves that are defined by TOMM40 genotype [70, 79]. The APOE4/4 curve would be unchanged, as the vast majority of $A P O E 4$ alleles carry the long (L) TOMM40 rs10524523 allele. There would be two curves for APOE3/4 individuals due to the presence of either a short (Sh) or a very long (VL) rs 10524523 polymorphism linked to $A P O E 3$. There would likely be three curves for $A P O E 3 / 3$ individuals due to the possible combination of alleles at rs 10524523 , i.e. short/short ( $\mathrm{Sh} / \mathrm{Sh})$, short/ very long $(\mathrm{Sh} / \mathrm{VL})$, and very long/very long $(\mathrm{VL} / \mathrm{VL})$

limited application as stand-alone predictive markers in healthy individuals prone to develop $\mathrm{MCI}$ and later $\mathrm{AD}$, and their utility in prevention studies is limited to monitoring changes over time during the course of the trial. These biomarkers are unlikely to serve as personalized indicators for early clinical intervention to prevent (or delay) incipient $\mathrm{AD}$ in and of themselves. In addition, the costs and complexity of these tools prohibit their utility in population-wide screening programs. By contrast, genetic tests are suitable for use as high-throughput populationwide screens, and have already been employed in a multitude of prevention programs, including those screening for newborn [82] and prenatal genetic disorders [83], various cancers [84, 85] and HIV drug-induced adverseevents [86]. The ease of sample collection (blood, saliva or buccal swabs), the stable nature of the information captured, and the low assay costs make genetic tests uniquely suitable for prediction and prevention applications. Moreover, genetic tests are widely used in routine clinical practice, therefore logistics of sample collection and testing, return of results and reimbursement infrastructure are in place. In principal, combining various types of biomarkers together with genetic indicators would likely yield the optimized positive and negative predictive value of $\mathrm{AD}$ disease onset, $\mathrm{MCI}$ conversion and related phenotypes. However, as outlined above, cost effectiveness and clinical utility analyses strongly favor diagnostics comprised of genetic and demographic data as the core test components in the vast majority of cases.

In addition to genetic involvement in the susceptibility to $\mathrm{AD}$ and its onset, a significant amount of work has been devoted to deciphering the genetic makeup associated with response to $\mathrm{AD}$ treatments. The study of genetic determinants associated with drug response phenotypes, including favorable versus insufficient response, variable pharmacokinetics and drug-induced adverse events, is termed pharmacogenetics [87, 88]. All currently approved treatments for $\mathrm{AD}$ offer temporary relief of some of the symptoms to merely some of the patients, but they do not arrest or alter the disease course. These drugs include three choline esterase inhibitors (ChEIs) for use in mild to moderate AD management. A fourth medication, an NMDA (N-methyl-D- aspartate) receptor antagonist, is approved for moderate to severe AD. Much research has thus been devoted in recent years to elucidating the role of acetylcholinesterase (AChE) and butyrylcholinesterase (BChE) in $\mathrm{AD}$ pathology and ChEI treatment response [89, 90]. Studies in the last decade, while overall inconclusive, report association of ChEI efficacy and safety with genetic determinants located primarily in $A P O E$ [91, 92] and the cytochrome P450 metabolizing enzyme gene CYP2D6 [93, 94]. In addition, several pharmaceutical development programs of disease modifying agents have reported response and/or safety stratification by $A P O E$ genotype, although results await validation. These include Wyeth/Elan's $A \beta$ antibody, bapineuzumab [95, 96]; GlaxoSmithKline's rosiglitazone [97]; and Accera's Ketasyn/Axona [98].

Understanding of the allele frequencies of variants highly associated with both $\mathrm{AD}$ risk/age-of-onset (such as markers in $A P O E$ and TOMM40) and/or pharmacogenetics (such as markers in $A P O E$ or CYP2D6) is especially important in the context of global clinical trials. Interpretation of the results of the trial without incorporation of pharmacogenetic data and ethnicity-specific allele frequency distribution could mask a successful outcome for a clinical trial, deeming it a failure [99].

\section{Concluding remarks and outlook}

The novel approach reviewed above utilizes phylogenetic methodology to collapse markers into the key polymorphisms that associate with complex human diseases. The key markers are then combined with epidemiological variables (e.g. age) to predict clinical outcomes. Once the analytical and clinical validity of the markers are confirmed, the diagnostics are tested prospectively for clinical utility in tandem with a phase-III prevention drug development program. In this fashion, trial results are designed to 
be directly translatable to healthcare guidance. Overall, this LOAD example serves as a ground-breaking implementation of the FDA's Rx/Dx co-development concept and paves the way for innovative application in other therapeutic areas, particularly complex disorders and drug response phenotypes with high estimates of heritability.

In conclusion, $\mathrm{AD}$ presents a pioneering example where research will lead to implementation of every aspect of predictive, preventive and personalized medicine. The majority of currently available biomarkers serve as tools during the investigation of disease progression, as well as during novel drug discovery and development. However, for the purpose of prediction and prevention the significant variables are the co-localized genetic markers in TOMM40 and $A P O E$, which account for the vast majority of variability in both risk and age-of-onset of the disease. In this fashion, the commonly accepted assumption that LOAD is underlined by a complex and elaborate set of genetic markers can potentially be refuted. The complexity can in fact be disentangled and reduced into a clear and minimal set of diagnostic markers. Moreover, an expedient path has been set forth to establish whether or not, and to what extent, these markers have clinical utility in supporting prevention therapy, paving the road for rational health management and development of insurance reimbursement programs. It is expected that this and similar approaches will lead to real personalization of care in $\mathrm{AD}$, as well as other medical conditions, for the benefit of patients, care givers and health systems globally.

Acknowledgements Supported by the Deane Drug Discovery Institute, Cabernet Pharmaceuticals, Inc., Shiraz Pharmaceuticals, Inc. and Zinfandel Pharmaceuticals, Inc. Additional support came from NIA grant AG028377. We thank the members of the Institute for Genomic Sciences and Policy (IGSP) and the Bryan Alzheimer's Disease Research Center (ADRC) at Duke University for their collaborative help.

Open Access This article is distributed under the terms of the Creative Commons Attribution Noncommercial License which permits any noncommercial use, distribution, and reproduction in any medium, provided the original author(s) and source are credited.

\section{References}

1. Matchar DB, Thakur ME, Grossman I, et al. Testing for cytochrome P450 polymorphisms in adults with non-psychotic depression treated with selective serotonin reuptake inhibitors (SSRIs). Evid Rep Technol Assess (Full Rep). 2007;146:1-77. PMID: 17764209.

2. ACCE model process for evaluating genetic tests. 2009. (Accessed at http://www.cdc.gov/genomics/gtesting/ACCE/index.htm.)

3. Hughes B. The comparative effectiveness challenge. Nat Rev Drug Discov. 2009;8(4):261-3. PMID.

4. Ginsburg G, SWillard HF. Genomic and personalized medicine: foundations and applications. Transl Res. 2009;154(6):277-87. PMID: 19931193.
5. FDA. Drug-diagnostic co-development concept paper (draft). In; 2005.

6. Hebert LE, Scherr PA, Bienias JL, et al. Alzheimer disease in the US population: prevalence estimates using the 2000 census. Arch Neurol. 2003;60(8):1119-22. PMID: 12925369.

7. National Institute Aging (2005-2006) Progress Report on Alzheimers Disease. PMID:

8. Brookmeyer R, Johnson E, Ziegler-Graham K, et al. Forecasting the global burden of Alzheimer's disease. Alzheimers Dement. 2007;3(3):186-91. PMID: 19595937.

9. Organization W H. The global burden of disease (2004 update). In. Geneva, Switzerland: WHO Press; 2004.

10. Blennow K, de Leon M, JZetterberg H. Alzheimer's disease. Lancet. 2006;368(9533):387-403. PMID: 16876668.

11. West RL. An application of prefrontal cortex function theory to cognitive aging. Psychol Bull. 1996;120(2):272-92. PMID: 8831298.

12. Visser PJ, Verhey FR. Mild cognitive impairment as predictor for Alzheimer's disease in clinical practice: effect of age and diagnostic criteria. Psychol Med. 2008;38(1):113-22. PMID: 17451628 .

13. Warfarin dosing. 2008. (Accessed at http://www.warfarindosing. org/Source/Home.aspx.)

14. Gatz M, Reynolds CA, Fratiglioni L, et al. Role of genes and environments for explaining Alzheimer disease. Arch Gen Psychiatry. 2006;63(2):168-74. PMID: 16461860.

15. Goate A, Chartier-Harlin MC, Mullan M, et al. Segregation of a missense mutation in the amyloid precursor protein gene with familial Alzheimer's disease. Nature. 1991;349(6311):704-6. PMID: 1671712.

16. Sherrington R, Rogaev EI, Liang Y, et al. Cloning of a gene bearing missense mutations in early-onset familial Alzheimer's disease. Nature. 1995;375(6534):754-60. PMID: 7596406.

17. Levy-Lahad E, Wasco W, Poorkaj P, et al. Candidate gene for the chromosome 1 familial Alzheimer's disease locus. Science. 1995;269(5226):973-7. PMID: 7638622.

18. Rogaev EI, Sherrington R, Rogaeva EA, et al. Familial Alzheimer's disease in kindreds with missense mutations in a gene on chromosome 1 related to the Alzheimer's disease type 3 gene. Nature. 1995;376(6543):775-8. PMID: 7651536.

19. Campion D, Dumanchin C, Hannequin D, et al. Early-onset autosomal dominant Alzheimer disease: prevalence, genetic heterogeneity, and mutation spectrum. Am J Hum Genet. 1999;65(3):664-70. PMID: 10441572.

20. Pericak-Vance MA, Grubber J, Bailey LR, et al. Identification of novel genes in late-onset Alzheimer's disease. Exp Gerontol. 2000;35(9-10):1343-52. PMID: 11113612.

21. Corder EH, Saunders AM, Strittmatter WJ, et al. Gene dose of apolipoprotein E type 4 allele and the risk of Alzheimer's disease in late onset families. Science. 1993;261(5123):921-3. PMID: 8346443.

22. Rocchi A, Pellegrini S, Siciliano G, et al. Causative and susceptibility genes for Alzheimer's disease: a review. Brain Res Bull. 2003;61(1):1-24. PMID: 12788204.

23. Bertram LTanzi RE. The genetic epidemiology of neurodegenerative disease. J Clin Invest. 2005;115(6):1449-57. PMID: 15931380.

24. Mahley RW. Apolipoprotein E: cholesterol transport protein with expanding role in cell biology. Science. 1988;240(4852):622-30. PMID: 3283935.

25. Strittmatter WJ, Saunders AM, Schmechel D, et al. Apolipoprotein E: high-avidity binding to beta-amyloid and increased frequency of type 4 allele in late-onset familial Alzheimer disease. Proc Natl Acad Sci U S A. 1993;90(5):1977-81. PMID: 8446617.

26. Bertram LTanzi RE. Thirty years of Alzheimer's disease genetics: the implications of systematic meta-analyses. Nat Rev Neurosci. 2008;9(10):768-78. PMID: 18802446. 
27. Farrer LA, Cupples LA, Haines JL, et al. Effects of age, sex, and ethnicity on the association between apolipoprotein E genotype and Alzheimer disease. A meta-analysis. APOE and Alzheimer Disease Meta Analysis Consortium. JAMA. 1997;278(16):134956. PMID: 9343467.

28. Roses AD. Apolipoprotein $\mathrm{E}$ affects the rate of Alzheimer disease expression: beta-amyloid burden is a secondary consequence dependent on APOE genotype and duration of disease. J Neuropathol Exp Neurol. 1994;53(5):429-37. PMID: 8083686.

29. Abraham R, Moskvina V, Sims R, et al. A genome-wide association study for late-onset Alzheimer's disease using DNA pooling. BMC Med Genomics. 2008;1:44. PMID: 18823527.

30. Li H, Wetten S, Li L, et al. Candidate single-nucleotide polymorphisms from a genomewide association study of Alzheimer disease. Arch Neurol. 2008;65(1):45-53. PMID: ISI:000252313000005.

31. Yu C-E, Seltman H, Peskind ER, et al. Comprehensive analysis of APOE and selected proximate markers for late-onset Alzheimer's disease: patterns of linkage disequilibrium and disease/marker association. Genomics. 2007;89(6):655-65. PMID.

32. Waring SC, Rosenberg RN. Genome-wide association studies in Alzheimer Disease. Arch Neurol. 2008;65(3):329-34. PMID

33. Beecham GW, Martin ER, Li YJ, et al. Genome-wide association study implicates a chromosome 12 risk locus for late-onset Alzheimer disease. Am J Hum Genet. 2009;84(1):35-43.

34. Waring SC, Rosenberg RN. Genome-wide association studies in Alzheimer disease. Arch Neurol. 2008;65(3):329-34. PMID: 18332245

35. Yu CE, Seltman H, Peskind ER, et al. Comprehensive analysis of APOE and selected proximate markers for late-onset Alzheimer's disease: patterns of linkage disequilibrium and disease/marker association. Genomics. 2007;89(6):655-65. PMID: 17434289.

36. Bu G. Apolipoprotein $\mathrm{E}$ and its receptors in Alzheimer's disease: pathways, pathogenesis and therapy. Nat Rev Neurosci. 2009;10 (5):333-44. PMID: 19339974.

37. Cedazo-Minguez A. Apolipoprotein E and Alzheimer's disease: molecular mechanisms and therapeutic opportunities. J Cell Mol Med. 2007;11(6):1227-38. PMID: 18205697.

38. Bazrgar M, Karimi M, Fathzadeh M, et al. Apolipoprotein E polymorphism in Southern Iran: E4 allele in the lowest reported amounts. Mol Biol Rep. 2008;35(4):495-9. PMID: 17594534.

39. Kok E, Haikonen S, Luoto T, et al. Apolipoprotein E-dependent accumulation of Alzheimer disease-related lesions begins in middle age. Ann Neurol. 2009;65(6):650-7. PMID: 19557866.

40. Mayeux R, Saunders AM, Shea S, et al. Utility of the apolipoprotein E genotype in the diagnosis of Alzheimer's disease. Alzheimer's Disease Centers Consortium on Apolipoprotein E and Alzheimer's Disease. N Engl J Med. 1998;338(8):50611. PMID: 9468467.

41. Cichon S, Craddock N, Daly M, et al. Genomewide association studies: history, rationale, and prospects for psychiatric disorders. Am J Psychiatry. 2009;166(5):540-56. PMID: 19339359.

42. Bertram L, McQueen MB, Mullin K, et al. Systematic meta-analyses of Alzheimer disease genetic association studies: the AlzGene database. Nat Genet. 2007;39(1):17-23. PMID: 17192785.

43. Lyssenko VGroop L. Genome-wide association study for type 2 diabetes: clinical applications. Curr Opin Lipidol. 2009;20(2):8791. PMID: 19276887.

44. McCarthy MI, Zeggini E. Genome-wide association studies in type 2 diabetes. Curr Diab Rep. 2009;9(2):164-71. PMID: 19323962.

45. Dickson SP, Wang K, Krantz I, et al. Rare variants create synthetic genome-wide associations. PLoS Biol. 2010;8(1):e1000294. PMID.

46. Goldstein DB. Common genetic variation and human traits. N Engl J Med. 2009;360(17):1696-8. PMID: 19369660.
47. Ioannidis JP, Thomas GDaly MJ. Validating, augmenting and refining genome-wide association signals. Nat Rev Genet. 2009;10(5):318-29. PMID: 19373277.

48. Coon KD, Myers AJ, Craig DW, et al. A high-density wholegenome association study reveals that APOE is the major susceptibility gene for sporadic late-onset Alzheimer's disease. J Clin Psychiatry. 2007;68(4):613-18. PMID: 17474819.

49. Potkin SG, Guffanti G, Lakatos A, et al. Hippocampal atrophy as a quantitative trait in a genome-wide association study identifying novel susceptibility genes for Alzheimer's disease. PLoS One. 2009;4(8):e6501. PMID: 19668339.

50. Harold D, Abraham R, Hollingworth P, et al. Genome-wide association study identifies variants at CLU and PICALM associated with Alzheimer's disease. Nat Genet. 2009;41 (10):1088-93. PMID: 19734902.

51. Lambert JC, Heath S, Even G, et al. Genome-wide association study identifies variants at CLU and CR1 associated with Alzheimer's disease. Nat Genet. 2009;41(10):1094-9. PMID: 19734903.

52. Heinzen EL, Need AC, Hayden KM et al. Genome-wide scan of copy number variation in late-onset Alzheimer's disease. J Alzheimers Dis. 2009. PMID: 19749422

53. Carrasquillo MM, Zou F, Pankratz VS, et al. Genetic variation in PCDH11X is associated with susceptibility to late-onset Alzheimer's disease. Nat Genet. 2009;41(2):192-8. PMID: 19136949.

54. Grupe A, Abraham R, Li Y, et al. Evidence for novel susceptibility genes for late-onset Alzheimer's disease from a genome-wide association study of putative functional variants. Hum Mol Genet. 2007;16(8):865-73. PMID: 17317784.

55. Reiman EM, Webster JA, Myers AJ, et al. GAB2 alleles modify Alzheimer's risk in APOE epsilon4 carriers. Neuron. 2007;54 (5):713-20. PMID: 17553421.

56. Bertram L, Lange C, Mullin K, et al. Genome-wide association analysis reveals putative Alzheimer's disease susceptibility loci in addition to APOE. Am J Hum Genet. 2008;83(5):623-32. PMID: 18976728.

57. Blass JP, Gibson GE. Cerebrometabolic aspects of delirium in relationship to dementia. Dement Geriatr Cogn Disord. 1999;10 (5):335-8. PMID: 10473934.

58. Davis JN, Hunnicutt EJ, Jr CJC. A mitochondrial bottleneck hypothesis of Alzheimer's disease. Mol Med Today. 1995;1 (5):240-7. PMID: 17607886.

59. Kessler J, Herholz K, Grond $\mathrm{M}$, et al. Impaired metabolic activation in Alzheimer's disease: a PET study during continuous visual recognition. Neuropsychologia. 1991;29(3):229-43. PMID: 2057055.

60. Blass JP. The mitochondrial spiral. An adequate cause of dementia in the Alzheimer's syndrome. Ann N Y Acad Sci. 2000;924:170 83. PMID: 11193795.

61. Castellani R, Hirai K, Aliev G, et al. Role of mitochondrial dysfunction in Alzheimer's disease. J Neurosci Res. 2002;70 (3):357-60. PMID: 12391597.

62. Manczak M, Anekonda TS, Henson E, et al. Mitochondria are a direct site of A beta accumulation in Alzheimer's disease neurons: implications for free radical generation and oxidative damage in disease progression. Hum Mol Genet. 2006;15(9):1437-49. PMID: 16551656.

63. Reddy PH, Beal MF. Amyloid beta, mitochondrial dysfunction and synaptic damage: implications for cognitive decline in aging and Alzheimer's disease. Trends Mol Med. 2008;14(2):45-53. PMID: 18218341.

64. Rui Y, Tiwari P, Xie Z, et al. Acute impairment of mitochondrial trafficking by beta-amyloid peptides in hippocampal neurons. $J$ Neurosci. 2006;26(41):10480-7. PMID: 17035532.

65. Devi L, Prabhu BM, Galati DF, et al. Accumulation of amyloid precursor protein in the mitochondrial import channels of human 
Alzheimer's disease brain is associated with mitochondrial dysfunction. J Neurosci. 2006;26(35):9057-68. PMID: 16943564.

66. Brodbeck J, Balestra ME, Saunders AM, et al. Rosiglitazone increases dendritic spine density and rescues spine loss caused by apolipoprotein E4 in primary cortical neurons. Proc Natl Acad Sci U S A. 2008;105(4):1343-6. PMID: 18212130.

67. Chang S, Ran Ma T, Miranda RD, et al. Lipid- and receptorbinding regions of apolipoprotein E4 fragments act in concert to cause mitochondrial dysfunction and neurotoxicity. Proc Natl Acad Sci U S A. 2005;102(51):18694-9. PMID: 16344479.

68. Anandatheerthavarada HK, Biswas G, Robin MA, et al. Mitochondrial targeting and a novel transmembrane arrest of Alzheimer's amyloid precursor protein impairs mitochondrial function in neuronal cells. J Cell Biol. 2003;161(1):41-54. PMID: 12695498.

69. Hansson Petersen CA, Alikhani N, Behbahani H, et al. The amyloid beta-peptide is imported into mitochondria via the TOM import machinery and localized to mitochondrial cristae. Proc Natl Acad Sci U S A. 2008;105(35):13145-50. PMID: 18757748.

70. Roses AD, Lutz MW, Amrine-Madsen $\mathrm{H}$ et al. A TOMM40 variable-length polymorphism predicts the age of late-onset Alzheimer's disease. Pharmacogenom J. 2009. PMID: 20029386.

71. Bekris LM, Millard SP, Galloway NM, et al. Multiple SNPs within and surrounding the apolipoprotein $\mathrm{E}$ gene influence cerebrospinal fluid apolipoprotein E protein levels. J Alzheimers Dis. 2008;13(3):255-66. PMID: 18430993.

72. Tachmazidou I, JDe VC, Iorio M. Genetic association mapping via evolution-based clustering of haplotypes. PLoS Genet. 2007;3 (7):e111. PMID: 17616979.

73. Frazer KA, Murray SS, Schork NJ, et al. Human genetic variation and its contribution to complex traits. Nat Rev Genet. 2009;10 (4):241-51. PMID: 19293820.

74. Wray GA. The evolutionary significance of cis-regulatory mutations. Nat Rev Genet. 2007;8(3):206-16. PMID: 17304246.

75. Biswas TK, Getz GS. Position-specific inhibition of yeast mitochondrial transcription by a poly(T) sequence. J Mol Biol. 1998;275(4):547-60. PMID: 9466930.

76. Beyer K, Humbert J, Ferrer A, et al. A variable poly-T sequence modulates alpha-synuclein isoform expression and is associated with aging. J Neurosci Res. 2007;85(7):1538-46. PMID: 17387688.

77. Hegde S, Lenox LE, Lariviere A, et al. An intronic sequence mutated in flexed-tail mice regulates splicing of Smad5. Mamm Genome. 2007;18(12):852-60. PMID: 18060457.

78. Chu CS, Trapnell BC, Curristin S, et al. Genetic basis of variable exon 9 skipping in cystic fibrosis transmembrane conductance regulator mRNA. Nat Genet. 1993;3(2):151-6. PMID: 7684646.

79. Lutz MW, Crenshaw DG, Saunders AM et al. Perspectives: genetic variation at a single locus and age of onset for Alzheimer's disease. Alzheimer's and Dementia. 2010. PMID:

80. Motter R, Vigo-Pelfrey C, Kholodenko D, et al. Reduction of betaamyloid peptide42 in the cerebrospinal fluid of patients with Alzheimer's disease. Ann Neurol. 1995;38(4):643-8. PMID: 7574461.

81. Jagust WJ, Landau SM, Shaw LM, et al. Relationships between biomarkers in aging and dementia. Neurology. 2009;73(15):11939. PMID: 19822868.

82. Fernhoff PM. Newborn screening for genetic disorders. Pediatr Clin North Am. 2009;56(3):505-13. Table of Contents. PMID: 19501689
83. Rosner G, Rosner SOrr-Urtreger A. Genetic testing in Israel: an overview. Annu Rev Genomics Hum Genet. 2009;10:175-92. PMID: 19453249.

84. Hahn NM, Kelley MR, Klaunig JE, et al. Constitutional polymorphisms of prostate cancer: prognostic and diagnostic implications. Future Oncol. 2007;3(6):665-82. PMID: 18041919.

85. Palomaki GE, Steinort K, Knight GJ, et al. Comparing three screening strategies for combining first- and second-trimester Down syndrome markers. Obstet Gynecol. 2006;107(2 Pt 1):36775. PMID: 16449126.

86. Lalonde RG, Thomas R, Rachlis A et al. Successful implementation of a national HLA-B*5701 genetic testing service in Canada. Tissue Antigens. 2009. PMID: 19843279.

87. Grossman I. Routine pharmacogenetic testing in clinical practice: dream or reality? Pharmacogenomics. 2007;8(10):1449-59. PMID: 17979518.

88. Roses AD. The medical and economic roles of pipeline pharmacogenetics: Alzheimer's disease as a model of efficacy and HLA-B $(*) 5701$ as a model of safety. Neuropsychopharmacology. 2009;34(1):6-17. PMID: 18923406.

89. Berson A, Knobloch M, Hanan M, et al. Changes in readthrough acetylcholinesterase expression modulate amyloid-beta pathology. Brain. 2008;131(Pt 1):109-19. PMID: 18056160.

90. Podoly E, Shalev DE, Shenhar-Tsarfaty S, et al. The butyrylcholinesterase $\mathrm{K}$ variant confers structurally derived risks for Alzheimer pathology. J Biol Chem. 2009;284(25):17170-9. PMID: 19383604.

91. Sjogren M, Hesse C, Basun $\mathrm{H}$, et al. Tacrine and rate of progression in Alzheimer's disease-relation to ApoE allele genotype. J Neural Transm. 2001;108(4):451-8. PMID: 11475012.

92. Choi SH, Kim SY, Na HR, et al. Effect of ApoE genotype on response to donepezil in patients with Alzheimer's disease. Dement Geriatr Cogn Disord. 2008;25(5):445-50. PMID: 18401173.

93. Cacabelos R, Llovo R, Fraile C, et al. Pharmacogenetic aspects of therapy with cholinesterase inhibitors: the role of CYP2D6 in Alzheimer's disease pharmacogenetics. Curr Alzheimer Res. 2007;4(4):479-500. PMID: 17908053.

94. Varsaldi F, Miglio G, Scordo MG, et al. Impact of the CYP2D6 polymorphism on steady-state plasma concentrations and clinical outcome of donepezil in Alzheimer's disease patients. Eur J Clin Pharmacol. 2006;62(9):721-6. PMID: 16845507.

95. Salloway S, Sperling R, Gilman S, et al. A phase 2 multiple ascending dose trial of bapineuzumab in mild to moderate Alzheimer disease. Neurology. 2009;73(24):2061-70. PMID: 19923550.

96. Kaufer D, Gandy S. APOE \{epsilon\}4 and bapineuzumab: Infusing pharmacogenomics into Alzheimer disease therapeutics. Neurology. 2009;73(24):2052-3. PMID: 19923549.

97. Risner ME, Saunders AM, Altman JF, et al. Efficacy of rosiglitazone in a genetically defined population with mild-tomoderate Alzheimer's disease. Pharmacogenomics J. 2006;6 (4):246-54. PMID: 16446752.

98. Costantini LC, Barr LJ, Vogel JL, et al. Hypometabolism as a therapeutic target in Alzheimer's disease. BMC Neurosci. 2008;9 Supp1 2:S16. PMID: 19090989.

99. Akkari PA, Swanson TW, Crenshaw DG, et al. Pipeline pharmacogenetics: a novel approach to integrating pharmacogenetics into drug development. Curr Pharm Des. 2009;15 (32):3754-63. PMID: 19925426. 